Editorial

\title{
Algorithm-Based Platelet Function Tests: A Need or a Greed?
}

\author{
Poonam Malhotra Kapoor ${ }^{1}$ \\ ${ }^{1}$ Department of Cardiac Anaesthesia, Cardiothoracic Centre, All \\ India Institute of Medical Sciences, New Delhi, India \\ J Card Crit Care TSS 2020;3:5-7
}

\section{Introduction}

Today, as the incidence of cardiovascular diseases increases, the use of antiplatelet therapy is widely recognized. This presents clinicians with the challenge of balancing the risk of thrombotic and bleeding complications. Platelet dysfunction is one of the chief causes of postoperative bleedings and their etiology is not fully understood. Platelets receptors point-of-care investigations are of a remarkable value in assessing patient's risk of bleeding. Reliable assessment of platelet function can improve treatment and saves lives.

Patients with triple-vessel coronary artery disease are usually advised to stop antiplatelet medication a few days prior to coronary artery bypass graft surgery (CABG) to reduce the incidence of postoperative bleeding. ${ }^{1}$ The patients whose antiplatelet drugs are not stopped, before CABG, are at greater risk of postoperative hemorrhage. ${ }^{2}$ As of 2019 , efforts are on to minimize blood transfusions. ${ }^{3}$ But, blood and blood products transfusion remains largely experiential and pragmatic. ${ }^{4}$ Hence, there is a need to have objective tests to demonstrate platelet dysfunction before platelet transfusion. Today, several devices perform platelet function tests which have been reported in clinical studies to evaluate platelet dysfunction and quantify the need for antiplatelet therapy. These devices range from highly sophisticated ones, such as light transmittance aggregometers and whole blood flow cytometers, to simple point-of-care technologies like the sonolcot, multiplate, thromboelastography (TEG), and thromboelastometry (ROTEM).

\section{Instruments Measuring Physical Properties of the Clot}

The balancing trial clot formation, retraction, and lysis reflects the ability of the hemostatic plug to perform its hemostatic function. Thus, by measuring various properties

Address for correspondence Poonam Malhotra Kapoor, MD, DNB, MNAMS, FIACTA (Hony), FTEE (Hony), FISCU (Hony), Department of Cardiac Anaesthesia, Cardiothoracic Centre, All India Institute of Medical Sciences, New Delhi, India (e-mail: drpoonamaiims@gmail.com). of the clot during hemostasis one can potentially detect several acquired and congenital platelet abnormalities. These instruments come with results in a shorter turnaround time.

The entire process of clot formation from its inception, retraction, and lysis can be well deciphered by the different signature curves seen graphically (based on the viscoelastic properties of blood) ( - Fig. 1a, $\mathbf{b})$.

Abnormal clot retraction may reflect deficiencies in platelet number, platelet glycoproteins, release of activating agents, or decreased fibrinogen levels. Several instruments have been developed that can accurately measure various physical properties of the clot with time. Examples include TEG, ROTEM, multiplate, and sonoclot.

The perplexing issue is that there is a hornets' nest regarding a few issues like: which device is optimal? What cutoff values to apply for discrimination of bleeding risk? How to proceed with antiplatelet strategies once the results of platelet testing are determined? No definitive guidelines exist in literature, on these.

\section{The Need for PFT: Necessity versus Cost?}

Platelet dysfunction may contribute to hemostatic alterations in cardiac surgery and can be influenced by preoperative antiplatelet therapy, comorbidities, or the effects of cardiopulmonary bypass itself. $^{5}$ Therefore, perioperative platelet function analysis has immense value in prediction of excessive bleeding and transfusion requirements and guiding hemostatic interventions.

When an agonist like arachidonic acid (ARA)/adenosine diphosphate (ADP)/thrombin-activating peptide (TRAP) (adenosine derivative $=$ ADP) is added to the blood sample, a signature curve is observed which measures platelet aggregation on the electrodes. A signature graph is then observed on the screen and the area under the curve (AUC), is a measure of this platelet aggregation. This AUC ( $\mathbf{- F i g . ~ 2 a , ~ b ) ~ i s ~ m e a s u r e d ~ i n ~ O h m s ~ a n d ~ i s ~ a ~ d i a g n o s t i c ~}$
Copyright @2020 Official Publication of The Simulation Society (TSS), accredited by International Society of Cardiovascular Ultrasound (ISCU).
License terms

(요 (1) $\Theta \circledast$
$10.1055 / \mathrm{s}-0039-3401443$

ISSN 2457-0206. 

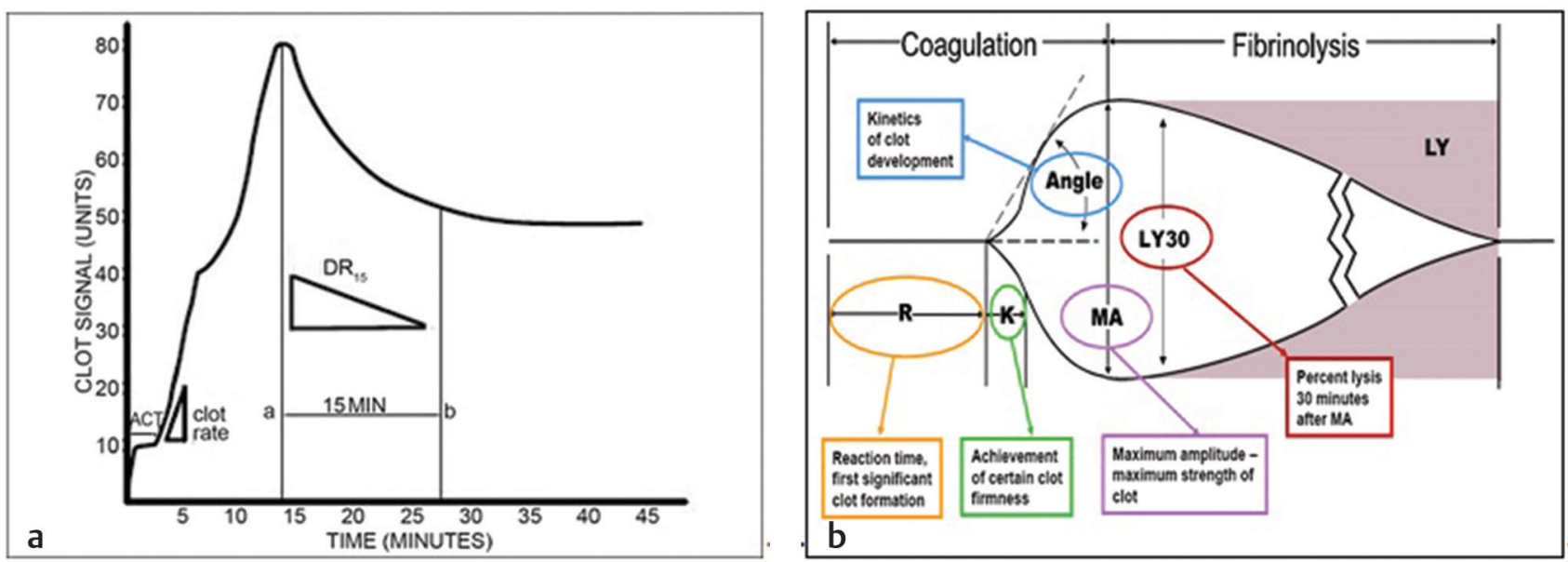

Fig. 1 (a, b) Typical sonoclot curve of normal hemostatis and normal thromboelastography (TEG).

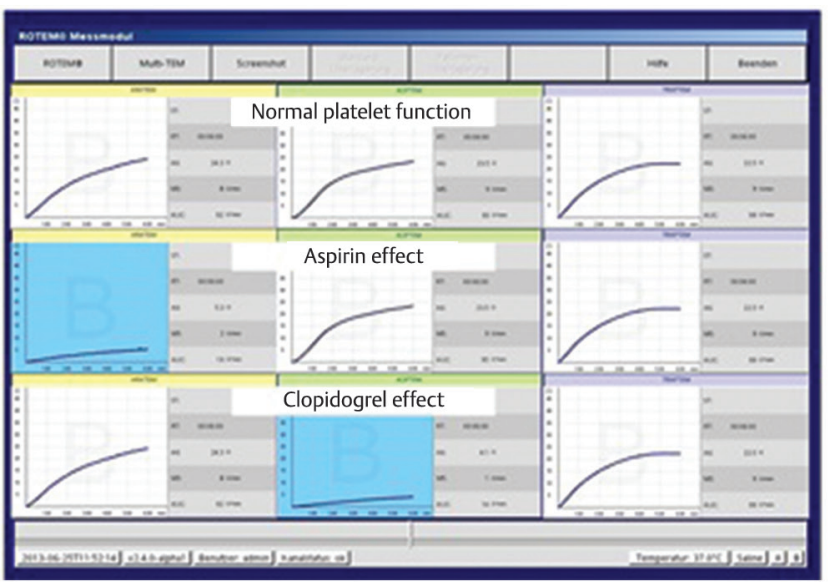

a

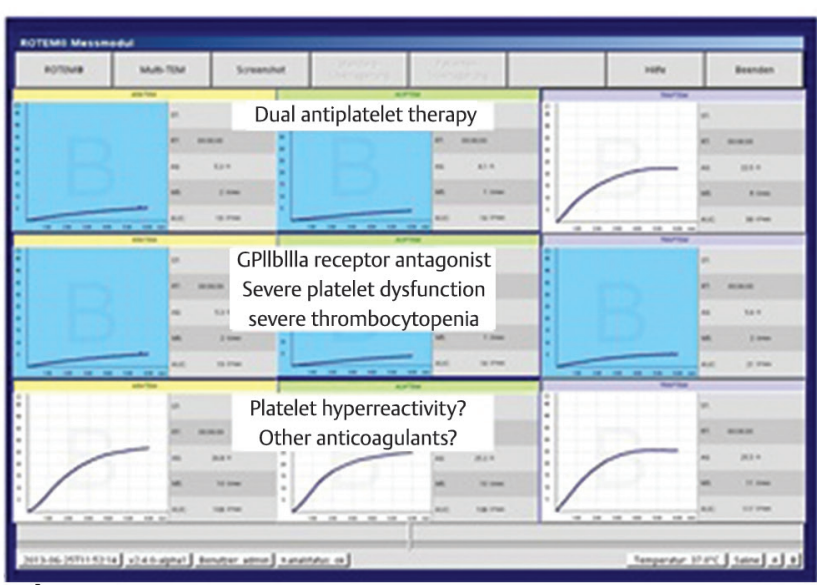

b

Fig. 2 (a, b) Showing platelet aggregometry graphs (on ROTEM platelet analyzer) for normal platelet function, aspirin clopidogrel, GP II b Illa thrombocyte, effects, and platelet hyperactivity curves.

number, signifying platelet receptor function. The multiplate electrode aggregometry (MEA) expressed in Ohms is presented as an aggregation unit (AU). The measured area under the graph (AUC, $1 \mathrm{U}=10 \mathrm{AU} \times \min$ ) has a diagnostic value and is now a clinical parameter defining the receptor function. ${ }^{6}$ Various studies have in recent time discussed PFA perioperative as a good prognostic marker.

Ranucci et al concluded that aggregometry testing can help in predicting postoperative bleeding in patients undergoing coronary artery bypass grafting.

In this special "AIIMS issue of JCCC" we are honored and privileged to have our Director AIIMS, a renowned pulmonologist and intensive care physician, write an editorial on "climate change and global warming: inhaling a lung attack." This is a visionary step for future predicaments of this epidemic social menace, especially in the Indian scenario.

Also seen in this issue in a brief communication by Gandhi et al is role of platelets in CABG patients. This is a class IIB evidence today in European hematology guidelines. Ameya et al, from our center, have emphasized on algorithm-based transfusion practice to prevent, perioperative bleeding in cyanotic babies. ${ }^{5}$ It makes for pensive reading about things to be seen in future when more patients on novel oral anticoagulants (NOACs) will be seen in the operating room and catheterization laboratory for invasive and noninvasive procedures. A platelet function analysis becomes mandatory then, as platelet count international normalized ratio (INR) and activated partial thromboplastin time (APTT) is not enough. It is in such situations that point of care tests with rapid turnaround time, in an algorithm-based manner, become need of the hour and a necessity. ${ }^{7}$ Objective platelet function assessment after cardiac surgery can predict postoperative blood loss, guide transfusion requirements, and discriminate the need for surgical re-exploration. Despite the cost, the life it saves makes it an important tool in the ICU and operating room. As endotheliosists we all have an important role to prevent bleeding when the endothelium is disrupted when patient is put on CPB (cardiopulmonary bypass)/PCI (percutaneous coronary intervention)/ECMP or VAD (ventricular assist device) insertions. 
Platelet aggregometry a point of care (POC) test indeed is a need which saves lives, only awareness of its availability and expertise in interpretations are required. It is not a luxury, despite its cost, as the rapidity with which it helps us in transfusing, the right blood component and save a life, is more important than the prolonged ICU ventilator stay. Its clinical utility and application remains to be discerned more.

An article by Sahu et al on Vitamin D toxicity is a must read.

\section{Conflict of Interest}

None.

\section{References}

1 Kozek-Langenecker SA, Afshari A, Albaladejo P, et al. Management of severe perioperative bleeding: guidelines from the European Society of Anaesthesiology. Eur J Anaesthesiol 2013;30(6):270-382

2 Di Dedda U, Ranucci M, Baryshnikova E, Castelvecchio S; Surgical and Clinical Outcome Research Group. Thienopyridines resistance and recovery of platelet function after discontinuation of thienopyridines in cardiac surgery patients. Eur J Cardiothorac Surg 2014;45(1):165-170

3 Vlot EA, Verwijmeren L, van de Garde EMW, Kloppenburg GTL, van Dongen EPA, Noordzij PG. Intra-operative red blood cell transfusion and mortality after cardiac surgery. BMC Anesthesiol 2019;19(1):65

4 Redfern RE, Fleming K, March RL, et al. Thrombelastography-directed transfusion in cardiac surgery: impact on postoperative outcomes. Ann Thorac Surg 2019;107(5):1313-1318

5 Sharan S, Magoon R, Karanjkar A, Kapoor PM. Utility of platelet function tests: a recent review round up. J Card Crit Care 2019;3(1):24-27

6 Ranucci M, Baryshnikova E, Soro G, Ballotta A, De Benedetti D, Conti D; Surgical and Clinical Outcome Research (SCORE) Group. Multiple electrode whole-blood aggregometry and bleeding in cardiac surgery patients receiving thienopyridines. Ann Thorac Surg 2011;91(1):123-129

7 Bhardwaj V, Kapoor PM, Karanjkar AA, et al. Ongenital cyanotic cardiac surgery in children: is algorithm-based pointof-care testing essential to prevent bleeding? J Card Crit Care 2018;2(2):84-90 